\title{
LA PHOTONIQUE DANS les industries agroalimentaires
}

\author{
Jacques COCHARD \\ TEMATYS \\ jcochard@tematys.com
}

\begin{abstract}
Par agro-industrie, on entend généralement la partie du secteur manufacturier qui transforme les matières premières et les demiproduits provenant du secteur agricole au sens large, c'est-à-dire y compris la foresterie et la pêche. Les méthodes optiques vont principalement se concentrer sur trois fonctions: la sécurité sanitaire, la qualité des produits, le contrôle des procédés. Chacune de ces fonctions obéit à des contraintes d'investissement et des logiques de marchés différentes, que l'on va analyser.
\end{abstract}

La qualité pour s'assurer dans des lots homogènes des paramètres organoleptiques (saveurs), mécaniques (craquant, fondant), et pour s'assurer en entrée/sortie de process de la conformité des produits.

À ces deux fonctions bien établies qui concernent les stocks de matières premières ou des produits transformés se rajoute une troisième fonction liée aux processus de transformation (contrôle temps-réel des procédés).

\section{Un marché de}

volume, dirivé part les règlementations: la sécurrité allimentaire

La sécurité alimentaire est un problème réglementaire avant tout. Des améliorations n'apparaissent sur le marché que quand les régulations sont disponibles et c'est le régulateur qui pilote ces modifications. Le seul argument marketing disponible pour le lancement d'un nouveau produit reste l'acceptation officielle et l'homologation de la méthode. La compétition est également très contrainte par les coûts (la boite de Pétri à quelques cents représente encore la référence pour de nombreux contrôles). Ces trois drivers (coût, homologation, adhésion aux méthodes éprouvées et aux standards) rendent ces marchés assez insensibles au temps (on peut toujours attendre un peu pour introduire une nouvelle méthode) et sont de ce fait largement occupés par des grands groupes de l'instrumentation comme Biomerieux.

Au niveau des besoins dans le domaine du contrôle sanitaire, l'Union européenne a mis au point suite à une demande forte des citoyens sur la traçabilité des produits, les ingrédients et matières premières utilisées dans les produits transformés, un système d'alerte RASFF $^{1}$ qui permet d'identifier, $d$ ' isoler à l'échelon du continent les divers problèmes alimentaires.

Ce système permet d'avoir une bonne perception des types de contamination et des produits agricoles ou agroalimentaires les plus porteurs de contamination et fait ressortir les enjeux majeurs dans le domaine (données 2015 extraites de http:/ ec.europa.eu/ food/safety/rasff/index_en.htm).

Par catégorie de contamination:

2 classes de contaminants (sur 25) représentent $20 \%$ des alertes - microorganismes pathogènes $(12 \%)$ et mycotoxines (8\%).

Par catégorie de produits agroalimentaires: 3 classes de produits (sur 32) représentent $23 \%$ des contaminations - fruits et légumes (10,2\%), noix et noisettes $(7,7 \%)$, poissons $(4,8 \%)$...

Food and Feed Safety Alert 
En termes de volumes de marchés, l'analyse microbiologique dans le domaine IAA est évaluée en 2015 à $1400 \mathrm{M} €$ pour 558 millions de tests ${ }^{2}$, en croissance en valeur de 4 à $6 \%$ les dernières années. Ces données témoignent d'un coût de test moyen de $2,6 €$, qui semble impossible à atteindre avec des méthodes optiques qui vont nécessiter un lecteur, un opérateur, du consommable incluant potentiellement des chimies de surface (dans le cas de la SPR ou du SERS) mais ce marché est en fait séparé en méthodes de routines classiques et en détection de pathogènes. Sur cette dernière application, on recherche des méthodes plus rapides, quand on ne peut pas disposer du temps nécessaire à la culture, avec des coûts pouvant aller vers $15 €$ voire $50 €$ sur des méthodes cytométriques, PCR, ELISA.

Sur ces dernières applications, des travaux d'optimisation des coûts sur des méthodes initialement dédiées à l'analyse biophysique permettent aujourd'hui d'envisager la commercialisation de méthodes de détection rapide de multiples bactéries pathogènes, en moins d'une journée, dans tout type de matrice alimentaire par résonance de plasmons de surface (SPR) et grâce à une biopuce fonctionnalisée avec des anticorps (PrestoDiag).

D'autres méthodes plus émergentes (cytométrie sans marquage) permettraient de détecter rapidement sans fluorophores, ni amplification, ni surface de contact, des bactéries dans un liquide par leur signature de diffraction (Hettich Lab Technology, licence de Purdue University) mais ces technologies restent encore en développement.

À titre d'exemple, sur l'ensemble des 558 millions de tests réalisés, $45 \%$ concernent la détection de pathogènes et dans cette catégorie, $22 \%$ environ concernent les contaminants chimiques (pesticides, mycotoxine). Sur ces 55 millions de test, les méthodes rapides en représentent $35 \%$ avec des coûts qui s'étagent entre

Référence : programmes européens GoodFood et Microsystems4Food
$15 €$ et $200 €$ principalement dans le domaine des mycotoxines (aflatoxin, patuline). Notons que pour ces prix, on recherche des seuils de détection à atteindre quasiment au niveau du diagnostic médical (20 pg/mL pour de l'aflatoxin 1), adaptés à des méthodes optiques telles que l'amplification Raman (SERS) ou la résonance plasmon (SPR).

\section{Des opportumités nouvelles dans le contrôle des procédés}

Nous avons indiqué précédemment les marges très faibles du secteur et la pression sur les prix exercée par la distribution et le consommateur luimême. La pression sur l'amont de la chaîne (producteurs agricoles) n'est plus à ce jour une politique responsable compte tenu du niveau de tension atteint dans le monde agricole. Dans cette optique (impossible de vendre plus cher, impossible d'acheter moins cher), tout outil de contrôle de la production pouvant réduire significativement - et à un coût maitrisé - la consommation inutile de produits bruts via une transformation mal conduite, et/ou éviter un niveau de saveur insuffisant en fin de procédés, et déclasser le produit lors de sa commercialisation, peut occuper un créneau dans cette industrie.

Jusqu'à très récemment, la photonique était pourtant restée en dehors du champ du contrôle procédé. On peut l'expliquer par trois facteurs.

\section{Des capacités d'investissement réduites sur des niches}

Il existe dans le domaine agroalimentaire une très large variété de procédés utilisés pour la transformation (fermentation, vinification, extrusion, irradiation...), une très grande diversité de produits à analyser (solides, liquides) et au sein d' un même segment (les fromages par exemple), une très grande variété de paramètres à analyser. Ces différents paramètres transforment un marché a priori de volume en une multitude de marchés de niches, et les capacités d'investissement déjà structurellement faibles deviennent sur certaines niches inexistantes. Or si la photonique donne une information riche, son développement pour une application donnée est coûteux.

\section{Une exploitation des données fournies qui reste complexe}

Par ailleurs, ces industries agroalimentaires, anciennes pour la plupart, ont le plus souvent mis au point des procédés basés sur des méthodes empiriques. L'industriel constate que le résultat est satisfaisant avec un nombre minimal de paramètre sous surveillance: température, $\mathrm{pH}$, salinité, pression, tous paramètres accessibles simplement par des méthodes non optiques. Et l'information supplémentaire apportée par les méthodes optiques demande des efforts de développement pour l'exploiter dans le pilotage du process. Les premières méthodes ont d'ailleurs été proposées par Ocean Optics (USA) pour mesurer optiquement des différentiels de $\mathbf{p H}$ pour le suivi de réaction au sein de cuves de fermentation.

\section{Des capacités en deçà des contraintes du marché}

Enfin, les méthodes classiques spectroscopiques (roues à filtres, spectrométrie FTIR ou par monochromateurs) qui mesurent les longueurs d'onde séquentiellement et avec des pièces mobiles, restaient peu adaptées à ces marchés où le débit et la robustesse des équipements est un paramètre clef.

L'arrivée en 2005-2010 de technologies qui permettent la mesure instantanée comme les matrices de diodes (Perten dans le groupe ThermoFischer) ou les bundles de fibres pour de la spectroscopie résolue spatialement (Indatech), a ouvert ce marché de la spectroscopie des procédés. Dans l'agroalimentaire, le marché est évalué en 2014 à plus de $150 \mathrm{M}$ \$ en croissance annuelle de 6,5\% avec des acteurs comme ABB, Agilent, Bruker, 
FOSS, ThermoFischer, Buchi, Horiba, Sartorius et constitue une importante zone de croissance pour les acteurs de la photonique.

\section{La qualité des produits, une profonde modification en cours dans le modèle d'activité}

L'ensemble du spectre est mis à contribution dans ce domaine. La vision industrielle a initialement permis de développer des solutions de contrôle de qualité des emballages, des étiquettes par vision industrielle avant de proposer des solutions pour le tri des produits agroalimentaires (Pellenc ST, Key Technologies...).

La spectroscopie NIR (proche-infrarouge) s'est ensuite développée dans ces domaines (FOSS puis Buchi, Thermo) pour apprécier la teneur en protéines et lipides des matières premières agricoles avant transformation. Des méthodes de fluorescence UV permettent également de détecter et quantifier des molécules très spécifiques comme les anthocyanes, polyphenols, et autres anti-oxydants (Force-A). Ces produits occupent à la fois les applications agricoles et agroindustrielles dans la mesure où les fabricants déclinent à présent des systèmes nomades de ces technologies, suffisamment performants compte tenu des spécificités des signatures à détecter.

L'arrivée de mesures temps réel par des caméras hyperspectrales VIS-NIR (Rikola), et les progrès très récents dans le domaine du terahertz tempsréel (Teratonics, Lytid) peuvent élargir le périmètre à des nouvelles applications (détection de contaminants organiques dans une matrice organique) que les méthodes actuelles (rayons X) ont des difficultés à réaliser.

Il s'agit là des marchés classiques du contrôle qualité. La qualité est ici l'affaire de l'industriel. Mais il y a également une défiance qui s'est établie entre consommateurs et producteurs («je ne fais confiance qu'à ce que je «vois»») en particulier depuis les révélations sur la nature des viandes incorporées dans les préparations. Aujourd'hui apparaissent de nombreux projets de spectroscopie nomade (sur smartphones) liés à la disponibilité de composants extrêmement compacts et peu chers, et à la possibilité de générer de la valeur hors du seul appareil, en développant des applications dédiées pour de très larges communautés d'utilisateurs.
Sur ces modèles d'activité impactés par la demande du consommateur, au contraire du domaine sanitaire, la constante de temps pour le déploiement des produits sera beaucoup plus courte. Ces nouveaux marchés seront beaucoup plus simples d'accès pour les PME et start-ups, comme on l'a vu dans le médical avec l'apparition du marché du «bien-être».

\section{Perspectives}

En conclusion, sur l'ensemble des trois métiers principaux dans l'agroalimentaire, la photonique a sa place à trouver pour des raisons différentes.

Dans le contrôle des pathogènes et des contaminants, les contraintes liées au temps de stockage des produits avant commercialisation, l'impact en termes d'image pour l'industriel lors d'une contamination microbienne, physique ou chimique contribuent à faire remonter le coût du test et à remettre les technologies optiques dans la compétition. Notons que les méthodes établies comme ELISA ou la PCR restent des méthodes rapides très bien installées avec pour la PCR des progrès importants dans le temps d'analyse, la capacité de multiplexage et l'automatisation des procédés.

\begin{tabular}{|c|c|c|c|c|c|}
\hline hazard category & alert & $\begin{array}{l}\text { border } \\
\text { rejection }\end{array}$ & $\begin{array}{l}\text { information } \\
\text { for attention }\end{array}$ & $\begin{array}{r}\text { information } \\
\text { for follow- } \\
\text { up }\end{array}$ & total \\
\hline adulteration / fraud & 1 & 89 & 3 & $\overline{6}$ & 99 \\
\hline allergens & 114 & 3 & 18 & 2 & 137 \\
\hline biocontarminants & 23 & 2 & 18 & 1 & 44 \\
\hline biotoxins (other) & 12 & & 5 & 1 & 18 \\
\hline chemical contamination (other) & 2 & & 2 & 4 & 8 \\
\hline composition & 51 & 19 & 22 & 26 & 118 \\
\hline food additives and flavourings & 17 & 55 & 32 & 36 & 140 \\
\hline foreign bodies & 43 & 23 & 14 & 30 & 110 \\
\hline GMO/ novel food & 4 & 18 & 3 & 20 & 45 \\
\hline heavy metals & 73 & 73 & 57 & 16 & 219 \\
\hline Industrial contaminants & 21 & 3 & 14 & 15 & 53 \\
\hline labelling absent/incomplete/incorrect & 6 & 8 & 3 & 9 & 26 \\
\hline migration & 14 & 38 & 12 & 13 & 77 \\
\hline mycotoxins & 74 & 388 & 29 & 4 & 495 \\
\hline non-pathogenic micro-organisms & 2 & 24 & 7 & 32 & 65 \\
\hline not determined / other & 5 & 5 & 1 & & 11 \\
\hline organoleptic aspects & & 25 & 3 & 10 & 38 \\
\hline packaging defective / incorrect & 5 & 6 & & 6 & 17 \\
\hline parasitic infestation & & 1 & 3 & 7 & 11 \\
\hline pathogenic micro-organisms & 261 & 265 & 136 & 83 & 745 \\
\hline pesticide residues & 24 & 292 & 71 & 18 & 405 \\
\hline poor or insufficient controls & 2 & 70 & 7 & 9 & 88 \\
\hline radiation & & 7 & 6 & 13 & 26 \\
\hline $\begin{array}{l}\text { residues of veterinary medicinal } \\
\text { products }\end{array}$ & 10 & 14 & 23 & 13 & 60 \\
\hline TSES & & & 2 & 19 & 21 \\
\hline
\end{tabular}

Alertes concernant les contaminations identifiées sur le territoire UE (2015) (source: RASFF).

Alertes concernant les produits contaminés identifiées sur le territoire UE (2015) (source: RASFF).

\begin{tabular}{|c|c|c|c|c|c|}
\hline product category & alert & $\begin{array}{l}\text { border } \\
\text { rejection }\end{array}$ & $\begin{array}{r}\text { information } \\
\text { for } \\
\text { attention }\end{array}$ & $\begin{array}{r}\text { information } \\
\text { for follow- } \\
\text { up }\end{array}$ & total \\
\hline alcoholic beverages & 4 & $\overline{1}$ & 1 & 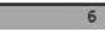 & 12 \\
\hline bivalve molluscs and products thereof & 23 & 7 & 28 & 3 & 61 \\
\hline cephalopods and products thereof & 1 & 15 & 2 & & 18 \\
\hline cereals and bakery products & 65 & 28 & 9 & 20 & 122 \\
\hline cocoa and cocoa preparations, coffee and tea & 12 & 32 & 7 & 7 & 58 \\
\hline compound feeds & 1 & & 2 & 18 & 21 \\
\hline confectionery & 12 & 10 & 4 & 7 & 33 \\
\hline crustaceans and products thereof & 5 & 26 & 19 & 9 & 59 \\
\hline dietetic foods, food supplements, fortified foods & 46 & 22 & 16 & 38 & 122 \\
\hline eggs and egg products & 7 & 3 & 2 & 2 & 14 \\
\hline fats and oils & 5 & 6 & 6 & 6 & 23 \\
\hline feed additives & & 1 & & 1 & 2 \\
\hline feed materials & 12 & 55 & 13 & 71 & 151 \\
\hline feed premixtures & & & & 2 & 2 \\
\hline fish and fish products & 104 & 67 & 88 & 38 & 297 \\
\hline food additives and flavourings & 1 & & & 6 & 7 \\
\hline food contact materials & 24 & 83 & 23 & 22 & 152 \\
\hline fruits and vegetables & 81 & 424 & 104 & 25 & 634 \\
\hline gastropods & & & & 3 & 3 \\
\hline herbs and spices & 40 & 74 & 30 & 6 & 150 \\
\hline honey and royal jelly & 1 & & 4 & 2 & 7 \\
\hline ices and desserts & 3 & & & 2 & 5 \\
\hline meat and meat products (other than poultry) & 83 & 24 & 33 & 19 & 159 \\
\hline milk and milk products & 48 & & 2 & 9 & 59 \\
\hline non-alcoholic beverages & 7 & 10 & & 9 & 26 \\
\hline nuts, nut products and seeds & 46 & 403 & 19 & 9 & 477 \\
\hline other food product / mixed & 11 & 16 & 2 & 5 & 34 \\
\hline pet food & 6 & 6 & 11 & 7 & 30 \\
\hline poultry meat and poultry meat products & 62 & 59 & 43 & 12 & 176 \\
\hline prepared dishes and snacks & 17 & 5 & 3 & 5 & 30 \\
\hline soups, broths, sauces and condiments & 20 & 3 & 3 & 9 & 35 \\
\hline wine & 3 & & 2 & & 5 \\
\hline
\end{tabular}


Dans le contrôle qualité et le contrôle des procédés, les progrès réalisés en termes de sensibilité dans les caméras NIR et la baisse des coûts permettent d'envisager de passer prochainement de la spectroscopie à de la spectro-imagerie dans la bande VIS-NIR. Statistiquement, et pour un grand nombre de productions, il apparaît en effet préférable de contrôler toute la production avec une moins bonne précision que les modalités actuelles avec un échantillonnage et un contrôle extrêmement précis. Aucune autre technologie ne peut permettre cette analyse temps réel de $100 \%$ de la production.

Dans le moyen infrarouge (MIR, au-delà de $2,5 \mu \mathrm{m}$ ), on voit les acteurs installés comme FOSS développer leur gamme de produits dans ces bandes spectrales qui autorisent la détection de composés invisibles dans les bandes NIR. Les sources QCL (lasers à cascade quantique) de plus en plus puissantes permettent d'intégrer des détecteurs moins sensibles conduisant à une baisse importante des coûts (prix des détecteurs et des sources). Une initiative comme la ligne pilote MIRPHAB, soutenue par la Commission européenne dans le cadre du Programme Public Privé photonique, autour d'acteurs nationaux comme MirSense, le CEA LETI, TEMATYS, ... se concentre sur le développement de modules adaptés à ces analyses dans le domaine du MIR, en complément et/ou remplacement des méthodes FTIR utilisées actuellement. La non-disponibilité de caméras dans le domaine du MIR avec la sensibilité requise ne permet toutefois pas d'envisager des méthodes de spectro-imagerie dans ces bandes à moyen terme.

Enfin, dans les bandes terahertz, la présence d'eau limite l'utilisation de cette gamme de fréquences à certaines

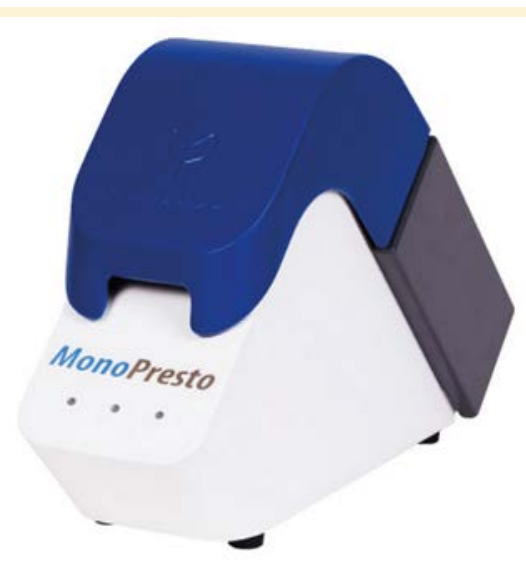

Lecteur MonoPresto réalisant des analyses de détection de pathogènes par SPR (Prestodiag).

applications (herbes sèches, produits gras). C'est surtout l'analyse des contenus d'emballages avant expédition qui semble prometteuse, car les matériaux d'emballage (carton, plastique, papier) sont en effet transparents à ces longueurs d'ondes.

\section{Your Application. Our Lasers.}
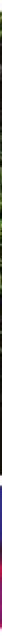

Coherent, Inc. is a Standard \& Poor's Small Cap 600 and a Russell 2000 Index company celebrating its 50th anniversary in 2016. Headquartered in Santa Clara, CA, USA, Coherent is a world leader in providing laser-based solutions to commercial and scientific research markets. We have the broadest technology portfolio in the industry with solutions for any application.

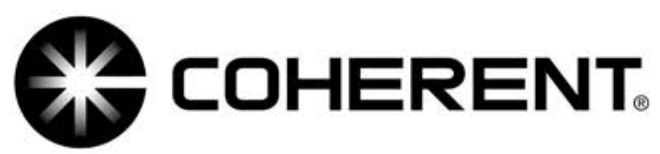

Superior Reliability \& Performance 